UT-771, IU-MSTP/19, hep-th/9704062

\title{
Duality in potential curve crossing: Application to quantum coherence
}

\author{
Kazuo Fujikawa \\ Department of Physics, University of Tokyo \\ Bunkyo-ku, Tokyo 113, Japan \\ Hiroshi Suzuki国 \\ Department of Physics, Ibaraki University, Mito 310, Japan
}

(September 6, 2018)

\begin{abstract}
A field dependent $s u(2)$ gauge transformation connects between the adiabatic and diabatic pictures in the (Landau-Zener-Stueckelberg) potential curve crossing problem. It is pointed out that weak and strong potential curve crossing interactions are interchanged under this transformation, and thus realizing a naive strong and weak duality. A reliable perturbation theory should thus be formulated in the both limits of weak and strong interactions. In fact, main characteristics of the potential crossing phenomena such as the Landau-Zener formula including its numerical coefficient are well-described by simple (time-independent) perturbation theory without referring to Stokes phenomena. We also show that quantum coherence in a double well potential is generally suppressed by the effect of potential curve crossing, which is
\end{abstract}


analogous to the effect of Ohmic dissipation on quantum coherence.

03.65.-w, 03.65.Sq, 31.15.-p, 32.80.Bx

Typeset using REVTEX 


\section{INTRODUCTION}

The potential curve crossing is related to a wide range of physical and chemical processes, and the celebrated Landau-Zener formula [1] 3] correctly describes the qualitative features of those processes [4]. An effort to improve the Landau-Zener formula and to make it a more quantitative formula is actively going on even recently [9,10]. The purpose of the present paper is to study this old problem on the basis of modern field theoretical ideas, namely, the duality and gauge transformation.

The adiabatic and diabatic pictures in potential curve crossing problem are related to each other by a field dependent $s u(2)$ gauge transformation [5, 8], and we point out that this transformation leads to an interchange of strong and weak potential curve crossing interactions, which is analogous to the electric and magnetic duality in conventional gauge theory [11]. This strong and weak duality allows a reliable perturbative treatment of potential curve crossing phenomena at the both limits of very weak (adiabatic picture) and very strong (diabatic picture) potential crossing interactions. Main features of potential curve crossing phenomena are well-described by straightforward time-independent perturbation theory combined with the zeroth order WKB wave functions [5, 8, 12], without referring to Stokes phenomena; perturbation theory thus becomes more flexible to cover a wide range of problems.

To illustrate our observation, we first show that simple time-independent perturbation theory gives an adequate description of the Landau-Zener formula including numerical coefficients in the both limits of adiabatic and diabatic pictures. We encounter an interesting topological object in the present formulation. We then apply our formulation to the analysis of the effect of potential curve crossing on quantum coherence in a double-well potential. We show that the potential crossing generally suppresses quantum coherence, which is analogous

to the effect of Ohmic dissipation on quantum coherence [13,14. To our knowledge, this clear recognition of duality and the precise criterion for naive perturbation theory including the analysis of quantum coherence have not been discussed before. 


\section{A MODEL HAMILTONIAN OF POTENTIAL CURVE CROSSING AND DUALITY}

To analyze the potential curve crossing, we start with a model Hamiltonian defined in the so-called diabatic picture [0, 8 ]

$$
H=\frac{1}{2 m} \hat{p}^{2}+\frac{V_{1}(x)+V_{2}(x)}{2}+\frac{V_{1}(x)-V_{2}(x)}{2} \sigma_{3}+\frac{1}{g} \sigma_{1}
$$

where $\sigma_{3}$ and $\sigma_{1}$ stand for the Pauli matrices. We assume throughout this article that the

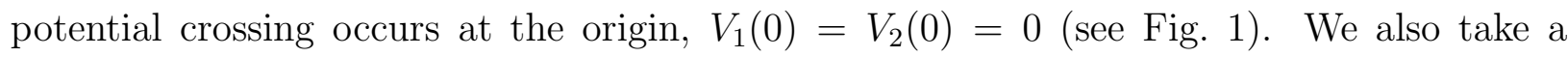
convention that the slope of the first potential at the crossing point is positive, $V_{1}^{\prime}(0)>$ 0 . The sign of $V_{2}^{\prime}(0)$ may be either the same as that of $V_{1}^{\prime}(0)$ (Section III) or opposite (Section IV).

If one neglects the last term in the above Hamiltonian, one obtains the unperturbed Hamiltonian in the diabatic picture

$$
H_{0} \equiv \frac{1}{2 m} \hat{p}^{2}+\frac{V_{1}(x)+V_{2}(x)}{2}+\frac{V_{1}(x)-V_{2}(x)}{2} \sigma_{3} .
$$

This Hamiltonian $H_{0}$ describes two potentials, which are decoupled from each other. The last term in (2.1), $H_{I} \equiv \sigma_{1} / g$ with a constant $g$, causes the transition between these two otherwise independent potential curves. In other words, if one takes $g \rightarrow$ large, this case physically corresponds to a complete potential crossing from a view point of adiabatic two-potential crossing (Fig. 2). Namely, $g$ stands for the strength of potential crossing interaction, and $g \rightarrow$ large corresponds to a very strong potential crossing interaction. On the other hand, if

one lets $g$ smaller, the effects of the last term in (2.1) become substantial and the Hamiltonian $H_{0}$ (2.2) does not present a sensible zeroth order Hamiltonian.

To deal with the case of a small $g$, we perform the non-Abelian "gauge transformation,"

$$
\Phi(x)=e^{i \theta(x) \sigma_{2} / 2} \Psi(x), \quad H^{\prime}=e^{i \theta(x) \sigma_{2} / 2} H e^{-i \theta(x) \sigma_{2} / 2},
$$

where $\sigma_{2}$ is a Pauli matrix. The Hamiltonian in the new picture is given by 


$$
\begin{aligned}
H^{\prime}= & \frac{1}{2 m}\left[\hat{p}-\frac{\hbar}{2} \partial_{x} \theta(x) \sigma_{2}\right]^{2}+\frac{V_{1}(x)+V_{2}(x)}{2} \\
& +\left[\frac{V_{1}(x)-V_{2}(x)}{2} \cos \theta(x)+\frac{1}{g} \sin \theta(x)\right] \sigma_{3} \\
& +\left[-\frac{V_{1}(x)-V_{2}(x)}{2} \sin \theta(x)+\frac{1}{g} \cos \theta(x)\right] \sigma_{1} .
\end{aligned}
$$

To eliminate the potential curve mixing, the last term of (2.4), we choose the gauge parameter $\theta(x)$ as 8

$$
\cot \theta(x)=g \frac{V_{1}(x)-V_{2}(x)}{2} \equiv f(x)
$$

We then obtain the Hamiltonian in the adiabatic picture

$$
H^{\prime}=H_{0}^{\prime}+H_{I}^{\prime}
$$

where

$$
H_{0}^{\prime} \equiv \frac{1}{2 m} \hat{p}^{2}+\frac{U_{1}(x)+U_{2}(x)}{2}+\frac{U_{1}(x)-U_{2}(x)}{2} \sigma_{3}
$$

and

$$
H_{I}^{\prime} \equiv-\frac{\hbar}{4 m}\left[\hat{p} \partial_{x} \theta(x)+\partial_{x} \theta(x) \hat{p}\right] \sigma_{2}+\frac{\hbar^{2}}{8 m}\left[\partial_{x} \theta(x)\right]^{2}
$$

The potential energies in the adiabatic picture are related to those in the diabatic picture as (Fig. 2)

$$
U_{1,2}(x) \equiv \frac{V_{1}(x)+V_{2}(x)}{2} \pm \sqrt{\left[\frac{V_{1}(x)-V_{2}(x)}{2}\right]^{2}+\frac{1}{g^{2}}}
$$

From the definition of the gauge parameter in (2.5), the "gauge field" $\partial_{x} \theta(x)$ is expressed as

$$
\partial_{x} \theta(x)=-\frac{f^{\prime}(x)}{1+f(x)^{2}} .
$$

The transition from the diabatic picture to the adiabatic picture is a local gauge transformation, or in the conventional field theoretical sense it is regarded as a field dependent transformation. The transformation from one of these two pictures to the other is an $x$ dependent notion. 
In the adiabatic picture, the $\sigma_{2}$ dependent term in the interaction $H_{I}^{\prime}$ (2.8) causes the potential crossing. If one neglects $H_{I}^{\prime}$, the two potentials characterized by $U_{1}(x)$ and $U_{2}(x)$ do not mix with each other: Physically, this means no potential crossing. This suggests that $H_{I}^{\prime}$ is proportional to the coupling constant $g$, since a small $g$ corresponds to weak potential crossing by definition. This is in fact the case as is clear from (2.10) and (2.5).

We thus conclude that the two extreme limits of potential crossing interaction should be reliably handled in perturbation theory; namely, the strong potential crossing interaction in the diabatic picture, and the weak potential crossing interaction in the gauge transformed adiabatic picture. This is analogous to the electric-magnetic duality in conventional gauge theory [11]: The diabatic picture may correspond to the electric picture with a coupling constant $e=1 / g$, and the adiabatic picture to the magnetic picture with a coupling constant $g$.

A general criterion for the validity of perturbation theory in the adiabatic picture (2.6) is

$$
\frac{\hbar}{2}\left|\partial_{x} \theta(x)\right| \ll|p(x)|
$$

which is expected to be satisfied when the coupling constant $g$ is small and the incident particle is sufficiently energetic.

\section{LANDAU-ZENER FORMULA}

As an application of the duality discussed in Section [I], we re-examine a perturbative derivation of the Landau-Zener formula in both of the adiabatic and diabatic pictures [1, 5, 8]. For definiteness, we shall assume $V_{1}^{\prime}(0)>V_{2}^{\prime}(0)$ as in Fig. 11.

Let us start with the adiabatic picture with weak potential crossing interaction. Since the gauge field generally vanishes, $\partial_{x} \theta(x) \rightarrow 0$ for $|x| \rightarrow \infty$, we can define the asymptotic states in terms of the eigenstates of $H_{0}^{\prime}(2.7)$. We define the initial and final states $\Phi_{i}$ and $\Phi_{f}$ by

$$
\Phi_{i}(x)=\left(\begin{array}{c}
\varphi_{1}(x) \\
0
\end{array}\right), \quad \Phi_{f}(x)=\left(\begin{array}{c}
0 \\
\varphi_{2}(x)
\end{array}\right)
$$


which satisfy

$$
\left[\frac{1}{2 m} \hat{p}^{2}+U_{1}(x)\right] \varphi_{1}(x)=E \varphi_{1}(x), \quad\left[\frac{1}{2 m} \hat{p}^{2}+U_{2}(x)\right] \varphi_{2}(x)=E \varphi_{2}(x) .
$$

We then obtain the potential curve crossing probability due to the perturbation $H_{I}^{\prime}(2.8)$

$$
w(i \rightarrow f)=\frac{2 \pi}{\hbar}\left|\left\langle\Phi_{f}\left|H_{I}^{\prime}\right| \Phi_{i}\right\rangle\right|^{2}
$$

The transition matrix element is given by

$$
\left\langle\Phi_{f}\left|H_{I}^{\prime}\right| \Phi_{i}\right\rangle=-\frac{\hbar^{2}}{4 m} \int_{-\infty}^{\infty} d x \partial_{x} \theta(x)\left[-\varphi_{2}^{\prime}(x) \varphi_{1}(x)+\varphi_{2}(x) \varphi_{1}^{\prime}(x)\right]
$$

To evaluate the matrix element, we use the WKB wave functions [四]:

$$
\varphi_{1}(x)= \begin{cases}\frac{C_{1}}{2 \sqrt{\left|p_{1}(x)\right|}} \exp \left[-\frac{1}{\hbar} \int_{a_{1}}^{x} d x\left|p_{1}(x)\right|\right] & \text { for } x>a_{1}, \\ \frac{C_{1}}{\sqrt{p_{1}(x)}} \cos \left[\frac{1}{\hbar} \int_{x}^{a_{1}} d x p_{1}(x)-\frac{\pi}{4}\right] & \text { for } x<a_{1},\end{cases}
$$

and

$$
\varphi_{2}(x)= \begin{cases}\frac{C_{2}}{2 \sqrt{\left|p_{2}(x)\right|}} \exp \left[-\frac{1}{\hbar} \int_{a_{2}}^{x} d x\left|p_{2}(x)\right|\right] & \text { for } x>a_{2}, \\ \frac{C_{2}}{\sqrt{p_{2}(x)}} \cos \left[\frac{1}{\hbar} \int_{x}^{a_{2}} d x p_{2}(x)-\frac{\pi}{4}\right] & \text { for } x<a_{2},\end{cases}
$$

where the semi-classical momenta in the adiabatic picture are defined by

$$
p_{1,2}(x) \equiv \sqrt{2 m\left[E-U_{1,2}(x)\right]}
$$

and $a_{1}$ and $a_{2}$ denote the classical turning points (Fig. 2). Within the WKB approximation, we also have

$$
\varphi_{1}^{\prime}(x) \simeq \begin{cases}-\frac{C_{1}}{2 \hbar} \sqrt{\left|p_{1}(x)\right|} \exp \left[-\frac{1}{\hbar} \int_{a_{1}}^{x} d x\left|p_{1}(x)\right|\right] & \text { for } x>a_{1}, \\ \frac{C_{1}}{\hbar} \sqrt{p_{1}(x)} \sin \left[\frac{1}{\hbar} \int_{x}^{a_{1}} d x p_{1}(x)-\frac{\pi}{4}\right] & \text { for } x<a_{1},\end{cases}
$$

and a similar relation for $\varphi_{2}^{\prime}(x)$. The normalization of $\varphi_{1}(x)$ is chosen as $C_{1}=2 \sqrt{m}$ to make the probability flux of the incident wave unity. On the other hand, the final state wave function in (3.3) has to be normalized by the delta function with respect to the energy, $\left\langle\Phi_{2}^{\prime} \mid \Phi_{2}\right\rangle=\delta\left(E_{2}^{\prime}-E_{2}\right)$ and this specifies $C_{2}=2 \sqrt{m} / \sqrt{2 \pi \hbar}$. 
We estimate the matrix element (3.4) by using the oscillating parts of the wave functions (3.5) and (3.6). This treatment is justified if the following conditions are satisfied:

(i) $|p(0)| \rightarrow$ large and $m \rightarrow$ large with $v=|p(0)| / m$ kept fixed such that non-relativistic treatment is valid in the physically relevant region.

(ii) $g \rightarrow$ small, but with

$$
\frac{1}{g} \ll \frac{1}{2 m}|p(0)|^{2}
$$

to ensure (2.11) and the condition

$$
\beta \ll a,
$$

where $a$ is an average turning point and $\beta$ is a typical geometrical extension of $\partial_{x} \theta(x)$. If (3.10) is satisfied, we can estimate the matrix element by using the oscillating parts of wave functions only since $\partial_{x} \theta(x)$ rapidly goes to zero for $|x| \gg \beta$ on the real axis.

The integral (3.4) is then written as

$$
\begin{aligned}
& \left\langle\Phi_{f}\left|H_{I}^{\prime}\right| \Phi_{i}\right\rangle \simeq-\frac{i \hbar C_{1} C_{2}}{8 m} \int d x \partial_{x} \theta(x) \\
& \times\left\{\exp \left[\frac{i}{\hbar} \int_{a_{1}}^{x} d x p_{1}(x)-\frac{i}{\hbar} \int_{a_{2}}^{x} d x p_{2}(x)\right]-\exp \left[-\frac{i}{\hbar} \int_{a_{1}}^{x} d x p_{1}(x)+\frac{i}{\hbar} \int_{a_{2}}^{x} d x p_{2}(x)\right]\right\}
\end{aligned}
$$

where we have set $p_{1}(x) / p_{2}(x)=1$ in the prefactors. This is justified within the saddle point approximation for $\hbar \rightarrow 0$; this is also justified if $\hbar /|p(0)| \ll \beta$, the characteristic length scale of the present problem, by letting $p(0)$ large as is specified in (i). Therefore we need to evaluate an integral of the form

$$
I \equiv \int_{-\infty}^{\infty} d x \partial_{x} \theta(x) \exp \left[\frac{i}{\hbar} \int_{a_{1}}^{x} d x p_{1}(x)-\frac{i}{\hbar} \int_{a_{2}}^{x} d x p_{2}(x)\right]
$$

We here present an explicit evaluation of (3.12) for the linear potential crossing problem, $V_{1}(x)=V_{1}^{\prime}(0) x$ and $V_{2}(x)=V_{2}^{\prime}(0) x$, on the basis of local data without referring to Stokes phenomena. For sufficiently large energy, $E-\left(U_{1}+U_{2}\right) / 2 \gg\left(U_{1}-U_{2}\right) / 2$, the difference of momenta can be approximated as [see (2.9)],

$$
\int_{0}^{x} d x\left[p_{1}(x)-p_{2}(x)\right] \simeq-\int_{0}^{x} d x \frac{2}{v(x) g \beta} \sqrt{x^{2}+\beta^{2}}
$$


where we used

$$
f(x)=g \frac{V_{1}^{\prime}(0)-V_{2}^{\prime}(0)}{2} x \equiv \frac{x}{\beta}, \quad v(x) \equiv \frac{1}{m} \sqrt{2 m\left[E-\frac{U_{1}(x)+U_{2}(x)}{2}\right]}
$$

and $v(x)$ is approximated to be a constant $v=v(0)$ in the following. We also have from (2.10)

$$
\partial_{x} \theta(x)=-\frac{\beta}{x^{2}+\beta^{2}}
$$

and thus

$$
\begin{aligned}
I & \simeq-\exp \left[\frac{i}{\hbar} \int_{a_{1}}^{0} d x p_{1}(x)-\frac{i}{\hbar} \int_{a_{2}}^{0} d x p_{2}(x)\right] \int_{-\infty}^{\infty} d x \frac{\beta}{x^{2}+\beta^{2}} \exp \left(-\frac{2 i}{\hbar v g \beta} \int_{0}^{x} d x \sqrt{x^{2}+\beta^{2}}\right) \\
& =-\exp \left[\frac{i}{\hbar} \int_{a_{1}}^{0} d x p_{1}(x)-\frac{i}{\hbar} \int_{a_{2}}^{0} d x p_{2}(x)\right] \int_{-\infty}^{\infty} d x \frac{1}{x^{2}+1} \exp \left(-i \alpha \int_{0}^{x} d x \sqrt{x^{2}+1}\right) \\
& =-\exp \left[\frac{i}{\hbar} \int_{a_{1}}^{0} d x p_{1}(x)-\frac{i}{\hbar} \int_{a_{2}}^{0} d x p_{2}(x)\right] \int_{-\infty}^{\infty} d x \exp [-i \alpha F(x)]
\end{aligned}
$$

where

$$
F(x) \equiv \int_{0}^{x} d x \sqrt{x^{2}+1}+\frac{1}{i \alpha} \ln \left(x^{2}+1\right), \quad \alpha \equiv \frac{2 \beta}{\hbar v g}=\frac{4}{\hbar v g^{2}\left[V_{1}^{\prime}(0)-V_{2}^{\prime}(0)\right]}>0 .
$$

We evaluate the integral (3.16) by a saddle point approximation with respect to $\alpha$. We thus seek the saddle point

$$
F^{\prime}(x)=\sqrt{x^{2}+1}+\frac{1}{i \alpha} \frac{2 x}{x^{2}+1}=0
$$

which is located between the real axis and the pole positions $x= \pm i$ of $\partial_{x} \theta(\beta x)$ so that we can smoothly deform the integration contour; these poles also coincide with the complex potential crossing points. If one sets $x=i y$ in (3.18) for $-1<y<1$, one has

$$
\sqrt{1-y^{2}}=-\frac{1}{\alpha} \frac{2 y}{1-y^{2}}
$$

which has a unique solution

$$
x_{s}=i y_{s} \simeq-i+\frac{i}{2}\left(\frac{2}{\alpha}\right)^{2 / 3}
$$

for large $\alpha$. (The complex conjugate of $x_{s}$ is located in the second Riemann sheet.) For this value of the saddle point 


$$
\begin{aligned}
& F\left(x_{s}\right)=\int_{0}^{x_{s}} d x \sqrt{x^{2}+1}+\frac{1}{i \alpha} \ln \left(\frac{2}{\alpha}\right)^{2 / 3} \simeq-\frac{\pi i}{4}+\frac{2}{3} \frac{i}{\alpha}+\frac{1}{i \alpha} \ln \left(\frac{2}{\alpha}\right)^{2 / 3} \\
& F^{\prime \prime}\left(x_{s}\right) \simeq-3 i\left(\frac{\alpha}{2}\right)^{1 / 3} .
\end{aligned}
$$

We thus have a Gaussian integral which decreases in the direction parallel to the real axis

$$
\begin{aligned}
I & \simeq-\left(\frac{\alpha}{2}\right)^{2 / 3} e^{2 / 3} \exp \left[\frac{i}{\hbar} \int_{a_{1}}^{0} d x p_{1}(x)-\frac{i}{\hbar} \int_{a_{2}}^{0} d x p_{2}(x)\right] \\
& \times \int_{-\infty}^{\infty} d x \exp \left[-3\left(\frac{\alpha}{2}\right)^{4 / 3}\left(x-x_{s}\right)^{2}\right] \exp \left(-\frac{\pi \alpha}{4}\right) \\
& =-\sqrt{\frac{\pi}{3}} e^{2 / 3} \exp \left[\frac{i}{\hbar} \int_{a_{1}}^{0} d x p_{1}(x)-\frac{i}{\hbar} \int_{a_{2}}^{0} d x p_{2}(x)\right] \exp \left(-\frac{\pi \alpha}{4}\right)
\end{aligned}
$$

From (3.11) we obtain

$$
\begin{gathered}
\left\langle\Phi_{f}\left|H_{I}^{\prime}\right| \Phi_{i}\right\rangle \simeq-\sqrt{\frac{\pi}{3}} e^{2 / 3} \sqrt{\frac{\hbar}{2 \pi}} \\
\sin \left\{\frac{1}{\hbar}\left[\int_{a_{1}}^{0} d x p_{1}(x)-\int_{a_{2}}^{0} d x p_{2}(x)\right]\right\} \\
\times \exp \left\{-\frac{\pi}{\hbar v g^{2}\left[V_{1}^{\prime}(0)-V_{2}^{\prime}(0)\right]}\right\} .
\end{gathered}
$$

It is interesting that the numerical value of the coefficient of the above expression, $\sqrt{\pi} e^{2 / 3} / \sqrt{3}=1.99317$, is very close to the canonical value 2 [4], and we replace it by 2 in the following. We thus have the transition probability from (3.3)

$$
\begin{aligned}
w(i \rightarrow f) & \simeq 4 \sin ^{2}\left\{\frac{1}{\hbar}\left[\int_{a_{1}}^{0} d x p_{1}(x)-\int_{a_{2}}^{0} d x p_{2}(x)\right]\right\} \exp \left\{-\frac{2 \pi}{\hbar v g^{2}\left[V_{1}^{\prime}(0)-V_{2}^{\prime}(0)\right]}\right\} \\
& \simeq 2 \exp \left\{-\frac{2 \pi}{\hbar v g^{2}\left[V_{1}^{\prime}(0)-V_{2}^{\prime}(0)\right]}\right\}
\end{aligned}
$$

where we replaced the square of sine function by its average $1 / 2$ in the final expression. We emphasize that the numerical coefficient of $w(i \rightarrow f)$ is fixed by perturbation theory and the local data without referring to global Stokes phenomena; this is satisfactory since linear potential crossing is a locally valid idealization.

We interpret that $w(i \rightarrow f)$ in (3.24) expresses twice the non-adiabatic transition probability. Notice that our initial state wave function contains the reflection wave as well as the incident wave. Therefore the transition probability per one crossing is given by the half of (3.24), 


$$
P(1 \rightarrow 2) \simeq \exp \left\{-\frac{2 \pi}{\hbar v g^{2}\left[V_{1}^{\prime}(0)-V_{2}^{\prime}(0)\right]}\right\},
$$

which is the celebrated Landau-Zener formula [4,5]. Our perturbative derivation presented here is conceptually much simpler than the past works [1, [4, 5, 8, [2], and it should be useful for a pedagogical purpose also.

It is interesting to study the same problem in the diabatic picture in Fig. [ 1 with $H_{I}=\sigma_{1} / g$ for large $g$. We first note that the initial state in the adiabatic picture asymptotically corresponds to the eigenfunction of $V_{2}(x)$ and the final state corresponds to $V_{1}(x)$, under the present setup $V_{1}^{\prime}(0)>V_{2}^{\prime}(0)$. Therefore the initial and final zeroth order wave functions in the diabatic picture are taken as

$$
\Psi_{i}(x)=\left(\begin{array}{c}
0 \\
\psi_{2}(x)
\end{array}\right), \quad \Psi_{f}(x)=\left(\begin{array}{c}
\psi_{1}(x) \\
0
\end{array}\right) .
$$

In the WKB approximation,

$$
\psi_{1}(x)= \begin{cases}\frac{C_{1}}{2 \sqrt{\left|p_{1}(x)\right|}} \exp \left[-\frac{1}{\hbar} \int_{a_{1}}^{x} d x\left|p_{1}(x)\right|\right] & \text { for } x>a_{1}, \\ \frac{C_{1}}{\sqrt{p_{1}(x)}} \cos \left[\frac{1}{\hbar} \int_{x}^{a_{1}} d x p_{1}(x)-\frac{\pi}{4}\right] & \text { for } x<a_{1},\end{cases}
$$

and

$$
\psi_{2}(x)= \begin{cases}\frac{C_{2}}{2 \sqrt{\left|p_{2}(x)\right|}} \exp \left[-\frac{1}{\hbar} \int_{a_{2}}^{x} d x\left|p_{2}(x)\right|\right] & \text { for } x>a_{2}, \\ \frac{C_{2}}{\sqrt{p_{2}(x)}} \cos \left[\frac{1}{\hbar} \int_{x}^{a_{2}} d x p_{2}(x)-\frac{\pi}{4}\right] & \text { for } x<a_{2} .\end{cases}
$$

In the above expressions, semi-classical momenta are defined by

$$
p_{1,2}(x) \equiv \sqrt{2 m\left[E-V_{1,2}(x)\right]} .
$$

The transition probability in the diabatic picture is then given by

$$
w(i \rightarrow f)=\frac{2 \pi}{\hbar}\left|\left\langle\Psi_{f}\left|H_{I}\right| \Psi_{i}\right\rangle\right|^{2} .
$$

The evaluation of the matrix element in (3.30) is the standard one described in the textbook of Landau and Lifshitz [4], for example. The saddle point for $\hbar \ll 1$ is located at the origin $x_{s}=0$ and we have an integral, for example, 


$$
\begin{aligned}
& \int_{-\infty}^{\infty} d x \frac{1}{\sqrt{p_{1}(x) p_{2}(x)}} \exp \left[\frac{i}{\hbar} \int_{a_{1}}^{x} d x p_{1}(x)-\frac{i}{\hbar} \int_{a_{2}}^{x} d x p_{2}(x)\right] \\
& \simeq \frac{\sqrt{2 \pi \hbar}}{\sqrt{m\left[V_{1}^{\prime}(0)-V_{2}^{\prime}(0)\right]}(2 m E)^{1 / 4}} \exp \left[\frac{i}{\hbar} \int_{a_{1}}^{0} d x p_{1}(x)-\frac{i}{\hbar} \int_{a_{2}}^{0} d x p_{2}(x)-\frac{\pi i}{4}\right] .
\end{aligned}
$$

Under this approximation, we have for $E>0$,

$$
\begin{aligned}
w(i \rightarrow f) & \simeq \frac{8 \pi}{\hbar v(0) g^{2}\left[V_{1}^{\prime}(0)-V_{2}^{\prime}(0)\right]} \cos ^{2}\left[\frac{1}{\hbar} \int_{0}^{a_{2}} d x p_{2}(x)-\frac{1}{\hbar} \int_{0}^{a_{1}} d x p_{1}(x)-\frac{\pi}{4}\right] \\
& \simeq \frac{4 \pi}{\hbar v g^{2}\left[V_{1}^{\prime}(0)-V_{2}^{\prime}(0)\right]}
\end{aligned}
$$

$[v(0)$ is the velocity at the crossing point, $v(0)=\sqrt{2 E / m}$. $]$ For $E<0$, one can verify that there is no saddle point and therefore,

$$
w(i \rightarrow f) \simeq 0
$$

We again interpret (3.32) as twice the potential crossing probability because our initial state wave function contains the reflection wave as well as the incident wave. The transition probability per one potential crossing is given by the half of (3.32).

A simple interpolating formula, which reproduces (3.24) in the weak coupling limit and (3.32) in the strong coupling limit, is given by

$$
w(i \rightarrow f) \simeq 2 \exp \left\{-\frac{2 \pi}{\hbar v g^{2}\left[V_{1}^{\prime}(0)-V_{2}^{\prime}(0)\right]}\right\}\left(1-\exp \left\{-\frac{2 \pi}{\hbar v g^{2}\left[V_{1}^{\prime}(0)-V_{2}^{\prime}(0)\right]}\right\}\right)
$$

This expression is also consistent with the (semi-classical) conservation of probability [4]. A more rigorous justification of (3.34) is facilitated by combining the analysis of Stokes phenomena and the conservation of probability [6].

Motivated by duality, we re-examined the perturbative derivation of the Landau-Zener formula, and we re-derived the formula (3.24) including its numerical coefficient on the basis of perturbation theory. However, our final formula (3.24) in the adiabatic picture does not contain the coupling constant as a prefactor. This is related to an interesting topological object in the present formulation. From the definition of (2.10), the "gauge field" satisfies the relation 


$$
\begin{aligned}
\int_{-\infty}^{\infty} d x \partial_{x} \theta(x) & =\theta(\infty)-\theta(-\infty) \\
& =-\pi
\end{aligned}
$$

which is independent of the coupling constant $g$; we assume $f(x) \rightarrow \pm \infty$ for $x \rightarrow \pm \infty$,

respectively. Because of this kink-like topological behavior of $\theta(x)$, the coupling constant does not appear as a prefactor of the matrix element in perturbation theory if the wave functions spread over the range which well covers the geometrical size of $\partial_{x} \theta(x)$. The precise criterion of the validity of perturbation theory is thus given by (2.11): This condition is in fact satisfied if the conditions (3.9)-(3.10) are satisfied. For small values of $x$, the small coupling $g$ helps to satisfy (2.11). Even for the values of $x$ near the average turning point $a$, we have

$$
\frac{\hbar}{2}\left|\partial_{x} \theta(a)\right| \simeq \frac{1}{2}\left(\frac{\beta}{a}\right) \frac{\hbar}{a} \ll \frac{\hbar}{a} \simeq|p(a)|,
$$

where $\beta$ stands for the typical geometrical size of $\partial_{x} \theta(x)$. The estimate in the left hand side is based on linear potentials (3.15), but we expect that the condition is satisfied for more general potentials as well.

To conclude this section, we clarified the basic mechanism why the prefactor of the Landau-Zener formula (3.25) should come out to be very close to unity in time-independent perturbation theory.

\section{LEVEL CROSSING AND QUANTUM COHERENCE}

The effects of dissipative interactions on macroscopic quantum tunneling have been extensively analyzed in the path integral formalism [13 and also in the canonical (field theoretical) formalism [14]. It is generally accepted that the Ohmic dissipation suppresses the macroscopic quantum coherence; in fact, an attractive idea of a dissipative phase transition has been suggested [13].

It is plausible that the effects of potential curve crossing with nearby potentials influence the quantum coherence of the two degenerate ground states. We here analyze the general 
features of the effects of potential crossing on quantum coherence on the basis of timeindependent perturbation theory, which was confirmed in the preceding section to be reliable in the both limits of weak and strong potential curve crossing interaction. We assume $V_{2}(x)=V_{1}(-x)$ and $V_{2}^{\prime}(0)<0$.

We thus analyze this problem from two different view points.

\section{A. Strong potential curve crossing interaction $g \gg 1$ (diabatic picture)}

We start with the diabatic picture in Fig. 3 and incorporate the effects of the potential crossing interaction $\sigma_{1} / g$. Namely, we (approximately) diagonalize the total Hamiltonian in the diabatic picture. By treating the last term of (2.1) as a perturbation for $g \gg 1$, we obtain the energy eigenvalues of the two lowest lying states as (with $E_{0}$ the zeroth order degenerate energy eigenvalue)

$$
E_{ \pm}^{(1)}=E_{0} \mp \frac{1}{g} \int_{-\infty}^{\infty} d x \psi_{L}(x) \psi_{R}(x)
$$

with the corresponding eigenfunctions

$$
\begin{aligned}
& \Psi_{+}(x) \simeq \frac{1}{\sqrt{2}}\left(\begin{array}{c}
\psi_{L}(x) \\
-\psi_{R}(x)
\end{array}\right) \equiv \frac{1}{\sqrt{2}}\left[e_{+} \psi_{L}(x)-e_{-} \psi_{R}(x)\right], \\
& \Psi_{-}(x) \simeq \frac{1}{\sqrt{2}}\left(\begin{array}{c}
\psi_{L}(x) \\
\psi_{R}(x)
\end{array}\right) \equiv \frac{1}{\sqrt{2}}\left[e_{+} \psi_{L}(x)+e_{-} \psi_{R}(x)\right] .
\end{aligned}
$$

Namely, both of these two states $\Psi_{ \pm}(x)$ choose

$$
\begin{aligned}
& e_{+} \psi_{L}(x) \text { in the region of left valley, } \\
& e_{-} \psi_{R}(x) \text { in the region of right valley. }
\end{aligned}
$$

The "spin" eigenstates $e_{ \pm}$and the "space" eigenstates $\psi_{L}(x)$ or $\psi_{R}(x)$ are strongly correlated. Note that one can conveniently think of the two potentials as spin-up and spin-down states. Originally, before we incorporate the perturbation $\sigma_{1} / g$ in (4.1), we have energetically degenerate two states with energy $E_{0}$ defined by 


$$
\Psi_{L}(x)=\left(\begin{array}{c}
\psi_{L}(x) \\
0
\end{array}\right) \equiv e_{+} \psi_{L}(x),
$$

which is always a spin-up state, and

$$
\Psi_{R}(x)=\left(\begin{array}{c}
0 \\
\psi_{R}(x)
\end{array}\right) \equiv e_{-} \psi_{R}(x),
$$

which is always a spin-down state.

In the strong potential crossing limit, $g \rightarrow \infty$, we have no effect of the conventional quantum tunneling in addition to the mixing in (4.2), since the conventional tunneling goes through the interaction $\sigma_{1} / g$ in (2.1), and thus higher order effects in $1 / \mathrm{g}$.

We can find the $O(1 / g)$ energy splitting in the WKB approximation. The left wave function $\psi_{L}(x)$ is given by (3.27) with $C_{1}=\sqrt{2 m \omega / \pi}$ (For a sufficiently low energy state such as the ground state, $\omega$ can be regarded as the curvature of the potential energy at the minimum.) The right wave function $\psi_{R}(x)$ is given by

$$
\psi_{R}(x)= \begin{cases}\frac{C_{2}}{2 \sqrt{\left|p_{2}(x)\right|}} \exp \left[-\frac{1}{\hbar} \int_{x}^{a_{2}} d x\left|p_{2}(x)\right|\right] & \text { for } x<a_{2}, \\ \frac{C_{2}}{\sqrt{p_{2}(x)}} \cos \left[\frac{1}{\hbar} \int_{a_{2}}^{x} d x p_{2}(x)-\frac{\pi}{4}\right] & \text { for } x>a_{2} .\end{cases}
$$

with $C_{2}=\sqrt{2 m \omega / \pi}$. In the saddle point approximation, we have the overlap integral

$$
\begin{aligned}
& \int d x \frac{1}{\sqrt{\left|p_{1}(x)\right|\left|p_{2}(x)\right|}} \exp \left[-\frac{1}{\hbar} \int_{a_{1}}^{x} d x\left|p_{1}(x)\right|+\frac{1}{\hbar} \int_{a_{2}}^{x} d x\left|p_{2}(x)\right|\right] \\
& \simeq \frac{\sqrt{2 \pi \hbar}}{\sqrt{m\left[V_{1}^{\prime}(0)-V_{2}^{\prime}(0)\right]}(-2 m E)^{1 / 4}} \exp \left[-\frac{1}{\hbar} \int_{a_{1}}^{0} d x\left|p_{1}(x)\right|+\frac{1}{\hbar} \int_{a_{2}}^{0} d x\left|p_{2}(x)\right|\right],
\end{aligned}
$$

which yields the energy splitting,

$$
\begin{aligned}
& E_{-}^{(1)}-E_{+}^{(1)} \\
& \simeq \frac{\sqrt{2 \hbar}}{g \sqrt{\pi\left[V_{1}^{\prime}(0)-V_{2}^{\prime}(0)\right]}}\left(\frac{m}{-2 E_{0}}\right)^{1 / 4} \exp \left\{-\frac{1}{\hbar} \int_{a_{1}}^{a_{2}} d x \sqrt{2 m\left[\bar{V}(x)-E_{0}\right]}\right\} .
\end{aligned}
$$

In the above expression, we have defined $\bar{V}(x)$ as the following " $\Lambda$-shape" potential:

$$
\bar{V}(x)= \begin{cases}V_{1}(x) & \text { for } x<0 \\ V_{2}(x) & \text { for } x>0 .\end{cases}
$$


We recall that, when the upper adiabatic potential $U_{1}(x)$ is absent, the standard WKB formula gives the energy splitting of lowest levels [4],

$$
E_{-}-E_{+} \simeq \frac{\hbar \omega}{\pi} \exp \left\{-\frac{1}{\hbar} \int_{-a}^{a} d x \sqrt{2 m\left[U_{2}(x)-E_{0}\right]}\right\}
$$

Comparing this with (4.8), we find that the quantum coherence is actually suppressed by two elements; the overall small coefficient $1 / g \ll 1$ and the exponential suppression factor; $\bar{V}(x)$ is always larger than the lower potential, $\bar{V}(x)>U_{2}(x)$.

\section{B. Weak potential curve crossing interaction $g \ll 1$ (adiabatic picture)}

In this case, we can use the gauge transformed adiabatic picture defined by (2.6). In this scheme, the low lying eigenstates for $H_{0}^{\prime}$ are described by the nearly degenerate two states in the lower potential curve $U_{2}(x)$. See Fig. 1 . For a small $g$, the two potential curves $U_{1}(x)$ and $U_{2}(x)$ are widely separated. We thus take the ground states of the spin-down sector (i.e., the nearly degenerate two ground states of the lower potential curve),

$$
\Phi_{+}(x)=\left(\begin{array}{c}
0 \\
\varphi_{2+}(x)
\end{array}\right), \quad \Phi_{-}(x)=\left(\begin{array}{c}
0 \\
\varphi_{2-}(x)
\end{array}\right)
$$

as the zeroth order approximation of the lowest lying states. These two states correspond to the conventional symmetric and anti-symmetric tunneling eigenstates of the double well potential $U_{2}(x)$, whose energy splitting gives the order parameter of the quantum coherence [13]. To the second order of the gauge field $\partial_{x} \theta(x)$, the energy eigenvalue is perturbed to

$$
\begin{aligned}
E_{ \pm}^{(2)}= & E_{ \pm}+\left\langle\Phi_{ \pm}\left|H_{I}^{\prime}\right| \Phi_{ \pm}\right\rangle-\sum_{n} \frac{\left|\left\langle\Phi_{1, n}\left|H_{I}^{\prime}\right| \Phi_{ \pm}\right\rangle\right|^{2}}{E_{1, n}-E_{ \pm}} \\
= & E_{ \pm}+\frac{\hbar^{2}}{8 m} \int d x\left[\partial_{x} \theta(x)\right]^{2} \varphi_{2 \pm}(x)^{2} \\
& -\frac{\hbar^{2}}{16 m^{2}} \sum_{n} \frac{1}{E_{1, n}-E_{ \pm}}\left|\int d x \varphi_{1, n}(x)\left[\hat{p} \partial_{x} \theta(x)+\partial_{x} \theta(x) \hat{p}\right] \varphi_{2 \pm}(x)\right|^{2}
\end{aligned}
$$

where $\Phi_{1, n}(x)$ [and $\varphi_{1, n}(x)$ ] is the $n$th energy eigenstate of the upper potential $U_{1}(x)$. 
It is generally difficult to conclude the suppression or enhancement of the quantum coherence solely from the perturbation formula (4.12). The reason is that the effect of intermediate state sum can depend on the details of the upper potential curve. Nevertheless, when the well of the upper potential is narrow enough, we can show that the quantum coherence is always suppressed.

We first assume that the lowest lying parity even and parity odd states are given respectively by a linear combination of the ground states in left and right wells:

$$
\varphi_{2 \pm}(x)=\frac{1}{\sqrt{2}}\left[\varphi_{2 L}(x) \pm \varphi_{2 R}(x)\right]
$$

where in the WKB approximation in the classically forbidden region,

$$
\varphi_{2 L}(x)=\varphi_{2 R}(-x)=\frac{C_{2}}{2 \sqrt{\left|p_{2}(x)\right|}} \exp \left[-\frac{1}{\hbar} \int^{x} d x\left|p_{2}(x)\right|\right] .
$$

The normalization constant is given by $C_{2}=\sqrt{2 m \omega / \pi}$.

We next assume that the upper potential $U_{1}(x)$ is narrow enough and can be well approximated by a square well potential with the width $a$. The eigenfunctions in the upper potential are therefore given by

$$
\varphi_{1, n}(x)=\sqrt{\frac{2}{a}} \sin \left[\frac{n \pi}{a}\left(x+\frac{a}{2}\right)\right], \quad n=1,2, \cdots
$$

Moreover, since the gauge field $\partial_{x} \theta(x)$ defined in $(2.10)$ is non-zero only within this narrow region, we may make the following replacements in the integral (4.12):

$$
\begin{aligned}
& \varphi_{2+}(x) \rightarrow \varphi_{2+}(0), \quad \varphi_{2-}(x) \rightarrow 0, \\
& \hat{p} \varphi_{2+}(x) \rightarrow 0, \quad \hat{p} \varphi_{2-}(x) \rightarrow i\left|p_{2}(0)\right| \varphi_{2+}(0),
\end{aligned}
$$

and

$$
\hat{p} \varphi_{1, n}(x) \rightarrow-i\left|p_{1, n}\right| \sqrt{\frac{2}{a}} \cos \left[\frac{n \pi}{a}\left(x+\frac{a}{2}\right)\right], \quad\left|p_{1, n}\right| \equiv \hbar \frac{n \pi}{a} .
$$

Under these assumptions, the energy shift (4.12) is estimated for the parity even state, 


$$
\begin{aligned}
E_{+}^{(2)}= & E_{+}+\frac{\hbar^{2}}{8 m} \int d x\left[\partial_{x} \theta(x)\right]^{2} \varphi_{2+}(0)^{2} \\
& -\frac{\hbar^{2}}{16 m^{2}} \sum_{n=1}^{\infty} \frac{\left|p_{1, n}\right|^{2}}{E_{1, n}-E_{+}}\left|\int d x \sqrt{\frac{2}{a}} \cos \left[\frac{n \pi}{a}\left(x+\frac{a}{2}\right)\right] \partial_{x} \theta(x)\right|^{2} \varphi_{2+}(0)^{2} .
\end{aligned}
$$

By noting $\left|p_{1, n}\right|^{2}=2 m\left[E_{1, n}-U_{1}(0)\right]$ and $U_{1}(0)>E_{+}$, we see that

$$
\frac{\hbar^{2}}{16 m^{2}} \frac{\left|p_{1, n}\right|^{2}}{E_{1, n}-E_{+}}=\frac{\hbar^{2}}{8 m} \frac{E_{1, n}-U_{1}(0)}{E_{1, n}-E_{+}}<\frac{\hbar^{2}}{8 m}
$$

and thus

$$
\begin{aligned}
E_{+}^{(2)}> & E_{+}+\frac{\hbar^{2}}{8 m} \int d x\left[\partial_{x} \theta(x)\right]^{2} \varphi_{2+}(0)^{2} \\
& -\frac{\hbar^{2}}{8 m} \sum_{n=0}^{\infty}\left|\int d x \sqrt{\frac{2}{a}} \cos \left[\frac{n \pi}{a}\left(x+\frac{a}{2}\right)\right] \partial_{x} \theta(x)\right|^{2} \varphi_{2+}(0)^{2} \\
= & E_{+},
\end{aligned}
$$

where we have added $n=0$ mode to make the cosine functions a complete set. Therefore the perturbation always increases the ground state energy.

On the other hand, eq. (4.12) gives for the parity odd state,

$$
E_{-}^{(2)}=E_{-}-\frac{\hbar^{2}}{16 m^{2}} \sum_{n=1}^{\infty} \frac{\left|p_{2}(0)\right|^{2}}{E_{1, n}-E_{-}}\left|\int d x \varphi_{1, n}(x) \partial_{x} \theta(x)\right|^{2} \varphi_{2+}(0)^{2} .
$$

Since the second term of (4.21) is negative definite, we see that the perturbation always lowers the odd state energy,

$$
E_{-}^{(2)}<E_{-}
$$

The perturbative corrections in (4.18) and (4.21) are proportional to the wave function at the origin, which satisfies $\varphi_{2+}(0)^{2} \simeq m\left(E_{-}-E_{+}\right) /\left[\hbar\left|p_{2}(0)\right|\right]$ [ 四], and therefore the correction to the energy splitting itself is proportional to the zeroth order energy splitting, $E_{-}-E_{+}$. Since the correction cannot excess the zeroth order value in a reliable region of perturbation theory, we should have $E_{-}^{(2)}-E_{+}^{(2)}>0$. By combining (4.20) and (4.22), therefore we find

$$
0<E_{-}^{(2)}-E_{+}^{(2)}<E_{-}-E_{+}
$$

which shows that the quantum coherence is suppressed. 
Although we derived the suppression of quantum coherence for the very special configuration in the weak coupling adiabatic picture, we expect that this suppression of quantum coherence is more general at least for small $g$. This is based on the following physical picture: When a particle tunnels the barrier in the lower potential curve, it has a small probability to cross to the upper potential curve. This potential curve crossing means that the particle enters deep inside the tunneling region of the upper potential curve and repelled back by the upper potential (as is expected in the diabatic picture), which in turn leads to the suppression of quantum tunneling of the particle. This argument leads to the general suppression of quantum coherence by potential crossing. This is also consistent with the suppression of barrier transmission in the scattering process by the non-adiabatic potential crossing effect [8].

Our explicit analyses both in the weak and strong potential crossing interactions suggest the general suppression of quantum coherence by potential crossing, which is analogous to the effect of Ohmic dissipation on quantum coherence [13,14]. In this connection, we may note that the formula (4.12) and the Ohmic dissipation in the Caldeira-Legget model 13] both correspond to the dipole approximation. However, an analysis of the basically nonperturbative tunneling effect in the second order perturbation theory needs a great care; for this reason, we were able to explicitly analyze the very specific case in (4.15)-(4.17). This suppression phenomenon of quantum coherence may become important in the future when one takes the effects of the environment into account in the analysis of potential curve crossing in physical and chemical processes.

\section{DISCUSSION AND CONCLUSION}

Motivated by the presence of interesting weak and strong duality in the model Hamiltonian (2.1) of potential curve crossing, we analyzed a perturbative approach to potential crossing phenomena. We have shown that straightforward perturbation theory combined with the zeroth order WKB wave functions provides a reliable description of general poten- 
tial crossing phenomena. Our analysis is based on the local data as much as possible without referring to global Stokes phenomena [12]. Formulated in this manner, perturbation theory becomes more flexible to cover a wide range of problems. From a perturbative view point, the treatment of the Landau-Zener formula in the adiabatic picture is most complicated since it does not contain the coupling constant in the prefactor. We pointed out that this absence of the coupling constant in the prefactor is related to the presence of an interesting kink-like topological object in the present formulation of adiabatic picture. The absence of the coupling constant in the prefactor is thus perfectly consistent with perturbation theory, provided that a more precise criterion of perturbation theory (2.11) is satisfied. In effect, we explained why the prefactor of the Landau-Zener formula should come out to be very close to unity in perturbation theory.

The suppression of quantum coherence by potential curve crossing, which to our knowledge has not been discussed before in this context, has also been neatly formulated in our treatment, in the both limits of very strong and very weak potential crossing interactions.

From a view point of general gauge theory, it is not unlikely that the electric-magnetic duality in conventional gauge theory is also related to some generalized form of potential crossing in the so-called moduli space [11]. We hope that our work may turn out to be relevant from this view point also.

Finally, we gratefully acknowledge H. Nakamura for critical comments which greatly helped improve the present work. 


\section{REFERENCES}

* $\quad$ electric mail: hsuzuki@mito.ipc.ibaraki.ac.jp

[1] L. D. Landau, Phys. Z. Sow. 1, 89 (1932); 2, 46 (1932).

[2] C. Zener, Proc. Roy. Soc. A137, 696 (1932).

[3] E. C. G. Stueckelberg, Helv. Phys. Acta. 5, 369 (1932).

[4] L. D. Landau and E. M. Lifshitz, Quantum Mechanics, translated by J. B. Sykes and J. S. Bell (Pergamon Press, 1977).

[5] M. S. Child, Molecular Collision Theory (Academic Press, 1974), and references therein.

[6] D. S. F. Crothers, Adv. Phys. 20, 406 (1971).

[7] J. B. Delos and W. R. Thorson, Phys. Rev. A6, 728 (1972); A9, 1026 (1974) (E).

[8] E. E. Nikitin and S. Ya. Umanskii, Theory of Slow Atomic Collisions (Springer-Verlag, 1984), and references therein.

[9] M. S. Child, Mol. Phys. 16 (1969) 313.

F. C. Carter, Molecular Electronic Devices (Marcel Dekker, 1982).

H. Nakamura, J. Chem. Phys. 97, 256 (1992).

[10] C. Zhu and H. Nakamura, J. Math. Phys. 33, 2697 (1992); J. Chem. Phys. 97, 1892 (1992); 102, 7448 (1995).

[11] P. A. M. Dirac, Proc. Roy. Soc. A133, 60 (1931).

C. Montonen and D. Olive, Phys. Lett. B72, 117 (1977).

N. Seiberg and E. Witten, Nucl. Phys. B426, 19 (1994).

[12] J. Heading, An Introduction to Phase-Integral Methods (Methuen and Co. Ltd., 1962). M. Froman and P. O. Froman, JWKB Approximation (North Holland, Amsterdam, 1965). 
M. S. Child (ed.), Semiclassical Methods in Molecular Scattering and Spectroscopy (D. Reidel, 1980).

V. P. Maslov and M. V. Fedoriuk, Semiclassical Approximation in Quantum Mechanics (D. Reidel, 1981).

W. H. Miller, Adv. Chem. Phys. 25, 69 (1974), and references therein.

[13] A. O. Caldeira and A. J. Legget, Phys. Rev. Lett. 46, 211 (1981); Ann. Phys. (N.Y.), 149, $374(1983)$.

A. J. Bray and M. A. Moore, Phys. Rev. Lett. 49, 1545 (1982).

S. Chakravarty, Phys. Rev. Lett. 49, 681 (1982).

G. Schoen and A. D. Zaikin, Phys. Rep. 198, 237 (1990), and references therein.

[14] K. Fujikawa, S. Iso, M. Sasaki and H. Suzuki, Phys. Rev. Lett. 68, 1093 (1992); Phys. Rev. B46, 10295 (1992). 


\section{FIGURES}

FIG. 1. Landau-Zener process in the diabatic picture.

FIG. 2. Landau-Zener process in the adiabatic picture.

FIG. 3. Quantum coherence in the diabatic picture.

FIG. 4. Quantum coherence in the adiabatic picture. 


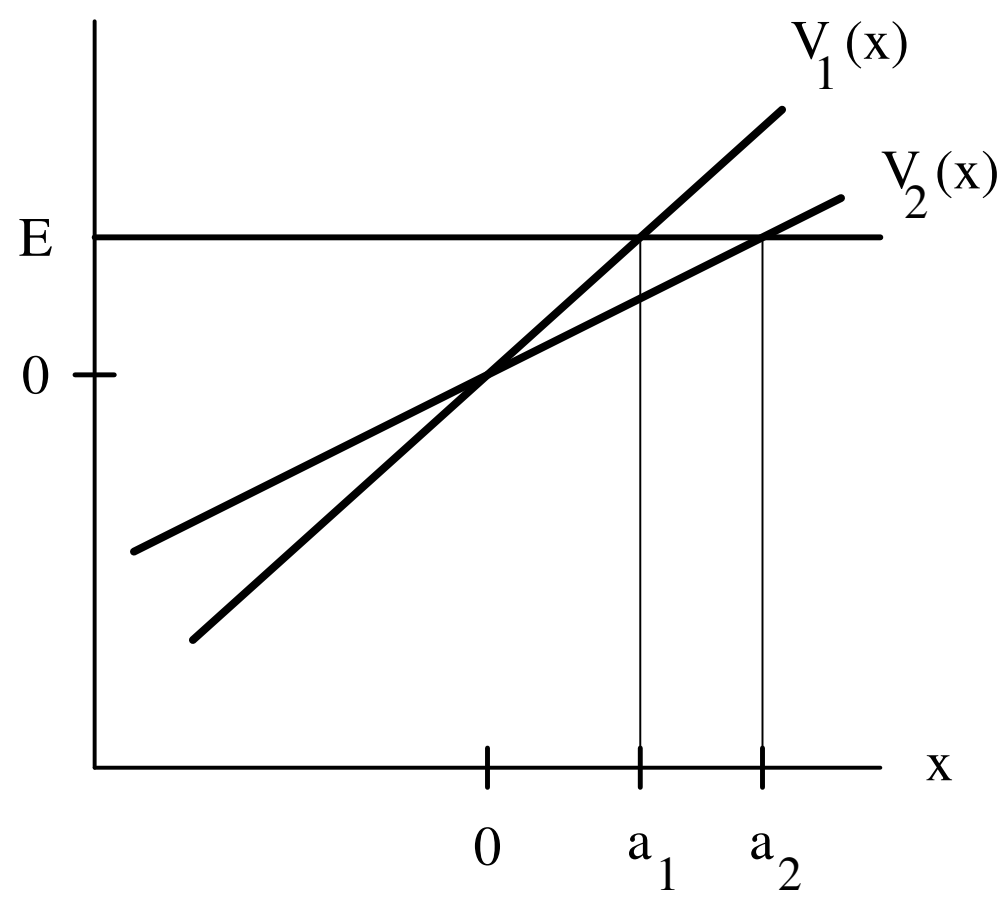

Fig. 1 


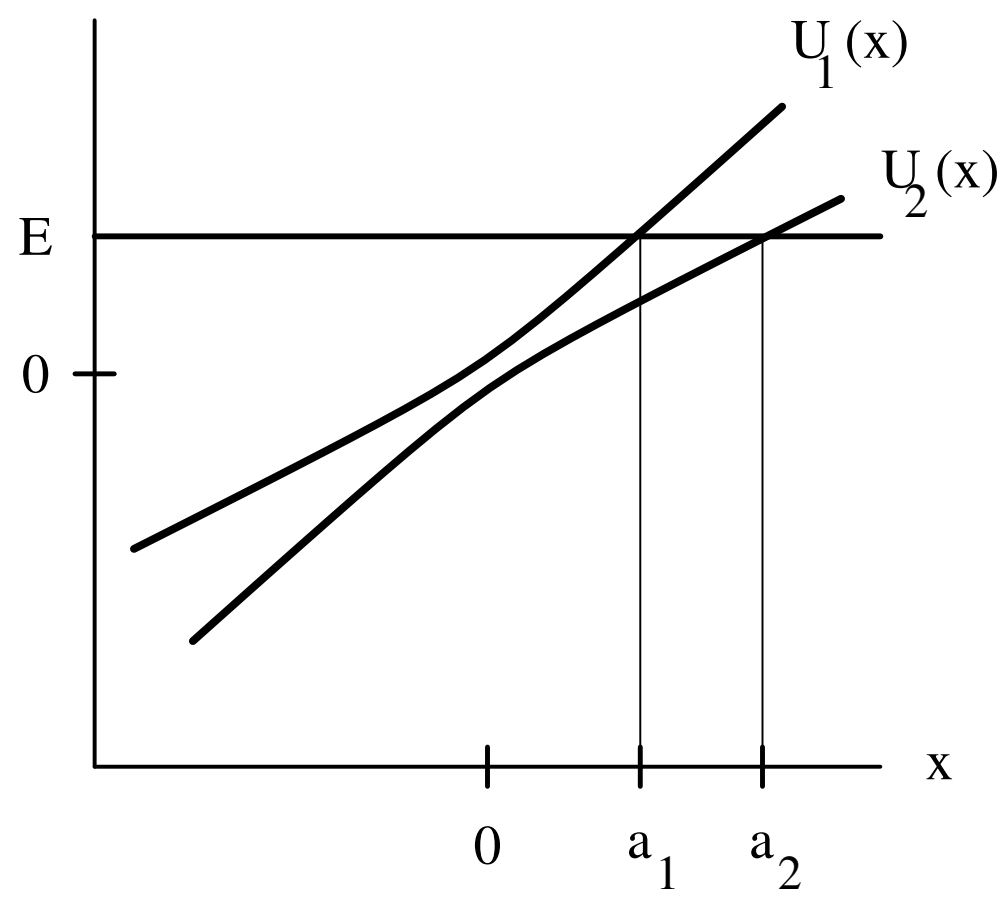

Fig. 2 


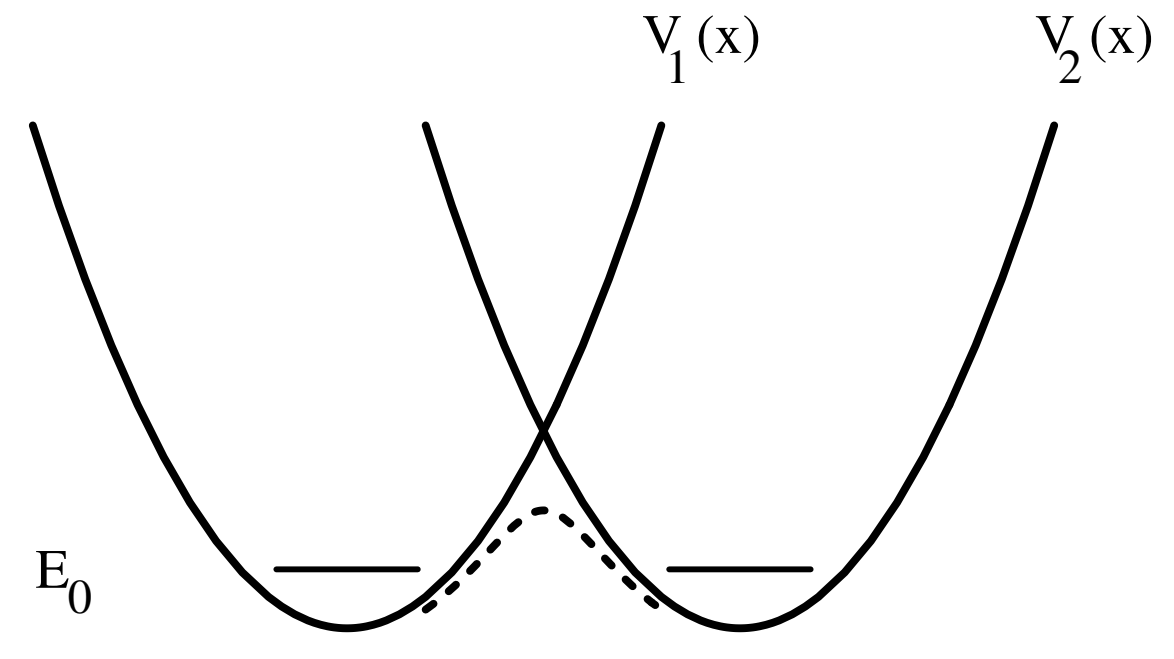

Fig. 3 


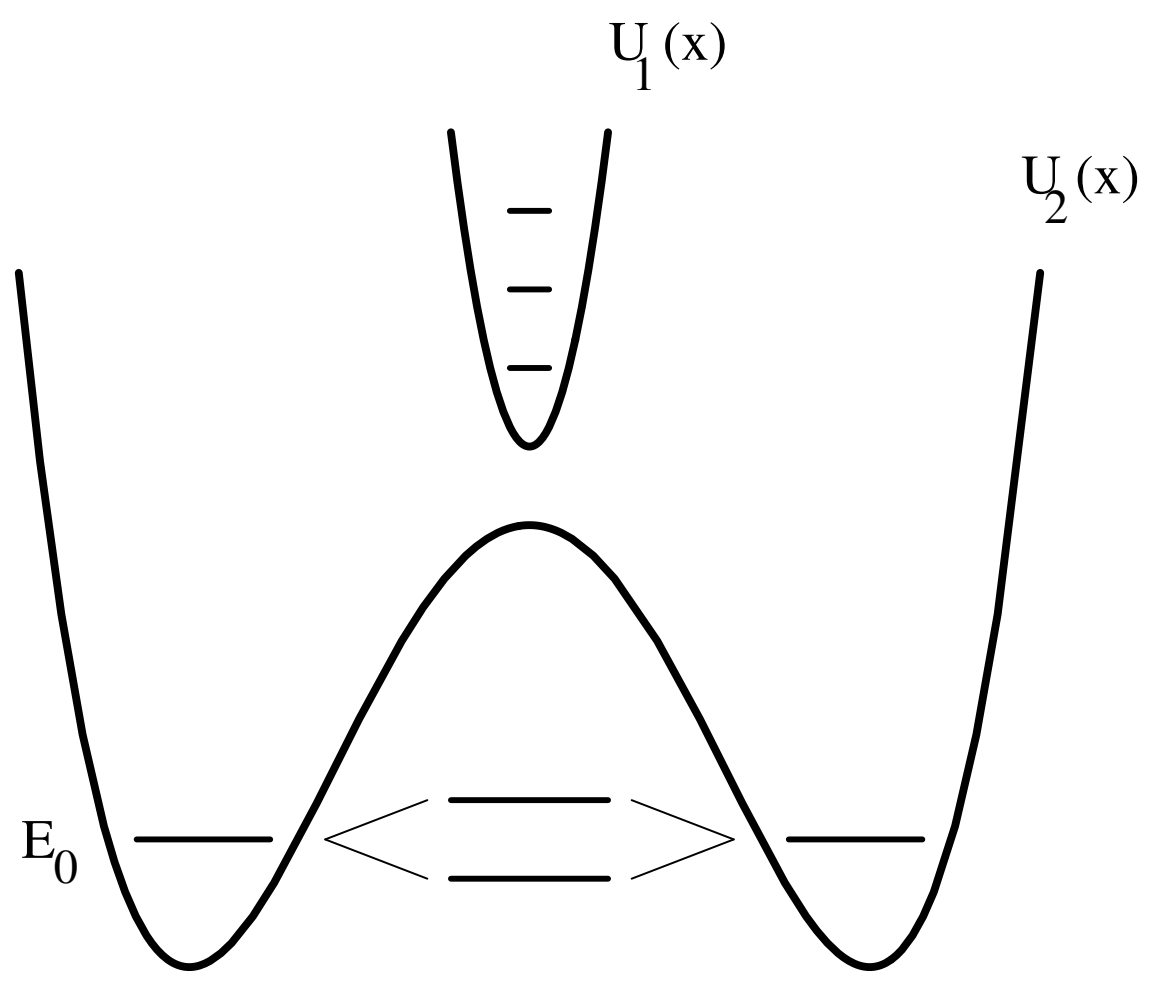

Fig. 4 